\title{
Evaluation of Nasal Functions While Wearing N95 Respirator and Surgical Facemask
}

\author{
Jianhua Zhu ${ }^{*}$, Shujin Lee², Deyun Wang ${ }^{3}$, Heow Pueh Lee ${ }^{1,4}$ \\ ${ }^{1}$ Department of Mechanical Engineering, National University of Singapore, Singapore City, Singapore \\ ${ }^{2}$ Department of Surgery, National University Health System, Singapore City, Singapore \\ ${ }^{3}$ Department of Otolaryngology, National University of Singapore, Singapore City, Singapore \\ ${ }^{4}$ National University of Singapore (Suzhou) Research Institute, Suzhou, China \\ Email: ${ }^{*}$ ihzhu.me@gmail.com
}

Received February 2014

\begin{abstract}
There is a lack of reported studies on how the long duration wearing of $\mathrm{N95}$ respirators or surgical facemasks will affect the upper airway functions. Considering the frequency of mask wearing especially in hospitals and during an outbreak of influenza, it is essential to have such data documented. Therefore, the current study is to establish the effect of long duration wearing of N95 and surgical facemasks on upper airway functions. 47 staffs of National University Hospital Singapore in 2013 were recruited. Each of the volunteers wore both $\mathrm{N} 95$ respirator and surgical facemask for 3 hours on two different days. During the period of mask wearing, relative airflow rates were recorded. Smell function test was carried out before and after mask wearing. The results show that no significant change of smell test score was found after removal of both the two types of masks. With N95 respirator, more air was breathed into the upper airways compared to surgical facemask.
\end{abstract}

\section{Keywords}

N95 Respirator, Surgical Facemask, Smell Function Test, Spirometry

\section{Introduction}

Facemasks are important components of personal protective equipment for medical personnel and workers in atmospherically-hostile environment. This is especially true for healthcare workers who need to interact with patients inflicted by airborne transmitted diseases such as the Severe Acute Respiratory Syndrome (SARS) outbreak that occurred in March 2003 [1]. Patients are also advised to put on facemasks in public areas to prevent the spread of airborne infectious diseases. Many national and international health agencies recommended the use of facemasks for the recent influenza A (H1N1) pandemic [2].

Many reported studies were done on the effectiveness of various facemasks in filtering out airborne pathogens, but very few of them focused on the discomfort level of their use. There were reported studies on the effects of

${ }^{*}$ Corresponding author. 
wearing N95 facemask and surgical facemasks on thermal stress and subjective sensations of the wearer [3]. Healthcare workers have to wear the facemasks for up to 12 hours and this may induce physiological stress on them [4]. In another reported study by Lim et al. [5], out of 212 healthcare workers who participated in the survey, $37.3 \%$ reported headaches when they wore the facemasks. Farmers wearing facemask while spraying pesticides in warm environment were also reported to experience heat stress on the body due to increased temperature and humidity within the facemask [6]. Although comfort level is subjective, there are a few parameters which can be used to correlate this comfort level. For example, a drier and cooler micro-climate leads to better comfort [3]. A higher expiratory and inspirational resistance reduces the ease of breathing and thus causes discomfort. A lower air flow volume means that the wearer may need to breathe in harder to get the same amount of fresh air required. In a recent study by Roberge et al. [7] using an automatic and metabolic simulator as a human surrogate, inhalation and exhalation resistances were found to increase by 0.43 and $0.23 \mathrm{~mm}$ of $\mathrm{H}_{2} \mathrm{O}$ pressure and it was concluded that increased exhaled moisture due to the wearing of facemask would not add significantly to the breathing resistance. However, the findings on surrogate were contrary to the common perception.

There is a lack of reported studies on how the long duration wearing of N95 respirators or surgical facemasks will affect the upper airway functions. The current study, therefore, is aimed to evaluate the effects of long during wearing of N95 respirators and surgical facemasks on upper airway functions such as smell function and airflow rate.

\section{Methodology}

Each of the participants would attend two experimental sessions. In the first session (S1), participants were requested to wear a N95 respirator (3M $\left.{ }^{\mathrm{TM}} 8210\right)$ for 3 hours, while in the second session (S2), participants were requested to wear a 3-ply surgical facemask (3M $\mathrm{M}^{\mathrm{TM}}$ earloop mask 1826). There are 63 participants involved in the first phase of this project, of which 5 participants were not qualified due to nasal diseases such as nasal polyp and rhinitis, 6 participants did not complete both of the 2 sessions and 5 other participants did not show up on the scheduled experimental date. 47 data sets were successfully collected, with 6 male subjects and 41 female subjects.

In the first 30 minutes of the two sessions, the participants were informed with details of the experiments, signed the consent form, and adapted to the room environment. Smell identification test was also done during this period as baseline of smell function. At the end of the first stage, the participants were told to put on the sensor of spirometry around the nostrils to measure the nasal airflow rate. In stage 2 (30 min to 3 hours $30 \mathrm{~min}$ ), the participants were told to put on either N95 respirator (first session) or surgical facemask (second session). Within this period, the airflow rates through nostrils were monitored. At the time of 3 hours 30 minutes, the masks were removed, and smell identification test was carried out again once the masks were removed. The RHINO-SYS (Happersberger otopront GmbH) was used to carry out the spirometry test. The smell identification test (Sensonics, Inc.) was used to measure the smell functions of the participants. In addition, the smell test was carried out only on the first 20 subjects and rescinded for the latter subjects, since no significant difference was found in the smell test scores between the results measured before and after wearing the masks.

\section{Results}

\subsection{Airflow Rate}

The Rhino-Move of Rhino-Sys (Happersberger otopront $\mathrm{GmbH}$ ) is a spirometer measuring the relative airflow rate passing through the nostrils during respiration. The relative airflow rate is obtained by recording the kinetic pressure fluctuations during respiration [8]. By extracting the recorded relative airflow rates at certain time points (every $15 \mathrm{~min}$ ) and averaging the airflow rates at these points among all the 47 subjects, the mean airflow rates at these points while wearing the masks are calculated. Figure 1 shows the averaged airflow rate of left and right airways at the 16 time points. In session S1, the airflow rate was generally larger in the right nasal airway in the first 2 hours compared to in the left nasal airway. In the last 1 hour of wearing N95 respirator, the airflow rates became comparable between the two airways. While during session S2, the airflow rate was comparable between the two airways through the whole 3 hours period. When adding up the airflow rates in the left and right airways, as shown in Figure 2, the relative respiratory airflow rates in nasal cavities during session S1 was generally larger than during session S2. 


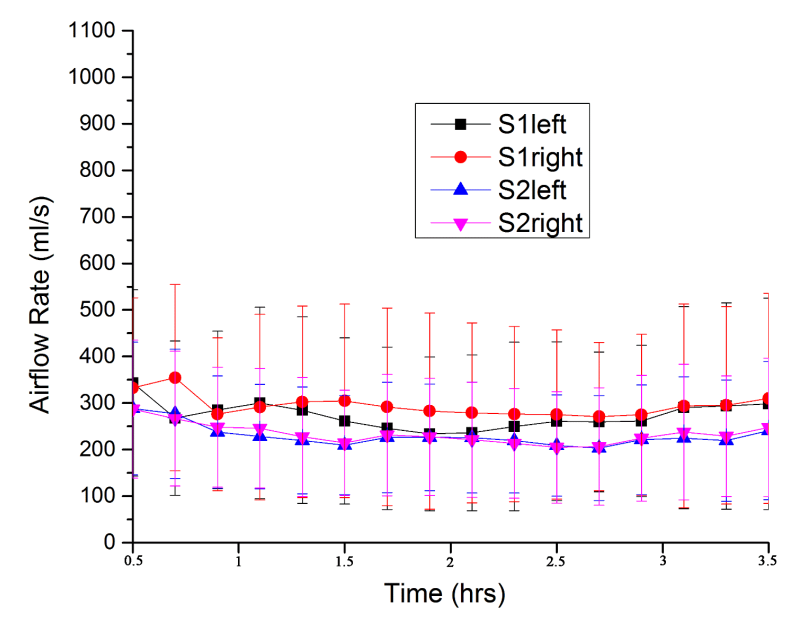

Figure 1. Averaged airflow rates during the 3 hours mask wearing period in sessions S1 and S2 through left and right nasal airways.

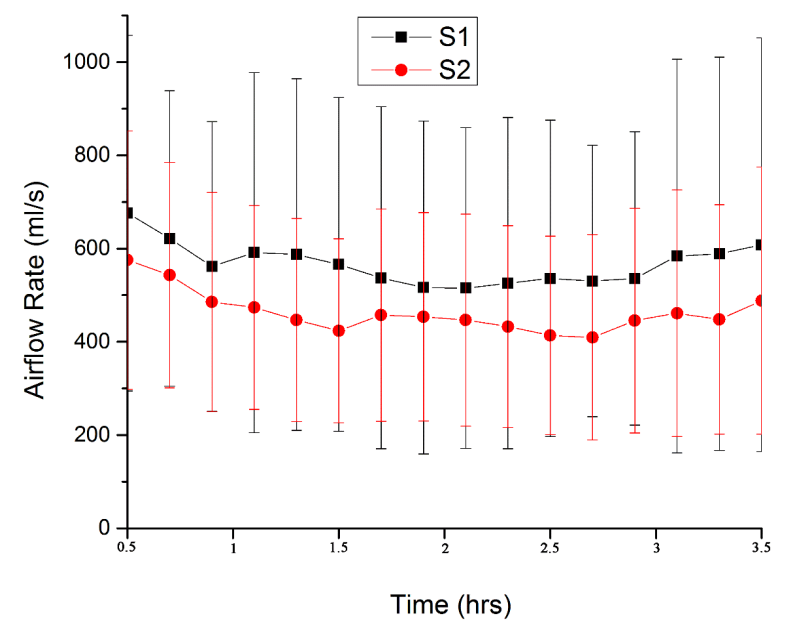

Figure 2. Averaged airflow rates during the 3 hours mask wearing period in sessions S1 and S2 through the whole nasal cavity.

By integrating the airflow rate with time during the 3 hours, the relative respired air volume is calculated with the equation below:

$$
V=\frac{\int_{0.5 \mathrm{hrs}}^{3.5 \mathrm{hrs}}\left(v_{l}+v_{r}\right) \mathrm{dt}}{2}
$$

where $V$ is the mean relative respired volume among the 47 subjects, $v_{1}$ and $v_{\mathrm{r}}$ are the airflow rates through the left and the right nostrils, respectively. The volumes of inspired air and expired air in one respiratory cycle are assumed to be equal. As shown in Figure 3, the mean volume of relative respired air during the 3 hours period is significantly larger in session S1 (2866.42 L) than in session S2 (2328.96 L).

\subsection{Smell Function}

Smell function test was done on 20 subjects before and after wearing the two types of masks in sessions $\mathrm{S} 1$ and S2. As shown in Table 1, the averaged score of smell identification test does not change much after wearing masks for 3 hours in both S1 and S2. 


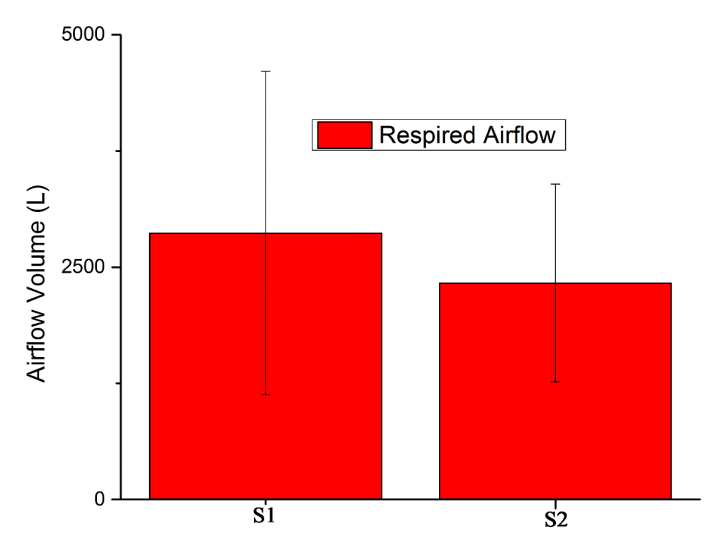

Figure 3. Averaged volume of air breathed into the nasal cavity during the 3 hours mask wearing period in sessions S1 and S2.

Table 1. Averaged score of smell identification test before and after wearing of the masks in sessions S1 and S2.

\begin{tabular}{ccc}
\hline & S1 & S2 \\
\hline Averaged Score Pre & $33.25( \pm 2.92)$ & $33.45( \pm 2.96)$ \\
Averaged Score Post & $33.20( \pm 2.76)$ & $33.10( \pm 3.41)$ \\
\hline
\end{tabular}

full mark: 40.

\section{Discussion}

The subjective ratings for perceptions on odorous while wearing the masks, reported by Li et al. [3], indicates that the smell functions might be different while the subjects wearing different types of masks. However, in the current study based upon objective smell identification test, no significant effect of wearing both N95 respirator and surgical facemask on nasal smell function was found. Since the averaged scores of smell function test are similar between the results measured for N95 respirator and surgical facemask, long-duration of mask wearing itself would not affect nasal smell function.

The chance for the particles such as bacteria penetrating the masks increases with airflow rate [9]. According to the present results, the relative airflow rate during respiration with masks on is significantly higher for N95 respirator compared to surgical facemask. However, since the N95 respirator is much more effective in filtering external particles than surgical facemask, the respirator is still a better choice.

\section{Conclusion}

Long-duration wearing of N95 respirator induced more air into the upper airways compared to surgical facemask. No significant effect of long duration wearing of both N95 respirator and surgical facemask was found on smell function.

\section{Acknowledgements}

The authors would like to acknowledge the support by the Academic Research Grant (CDPHRG/12NOV001) from the Ministry of Health, Singapore. This research has been approved by the relevant Institutional Review Board.

\section{References}

[1] Seto, W.H., Tsang, D., Yung, R.W.H., Ching, T.Y., Ng, T.K., Ho, M., Ho, L.M. and Peiris, J.S.M. (2003) Effectiveness of Precautions against Droplets and Contact in Prevention of Nosocomial Transmission of Severe Acute Respiratory Syndrome (SARS). The Lancet, 361, 1519-1520. http://dx.doi.org/10.1016/S0140-6736(03)13168-6 
[2] Cowling, B.J., Zhou, Y., Ip, D.K.M., Leung, G.M. and Aiello, A.E. (2010) Face Masks to Prevent Transmission of Influenza Virus: A Systematic Review. Epidemiology \& Infection, 138, 449-456. http://dx.doi.org/10.1017/S0950268809991658

[3] Li, Y., Tokura, H., Guo, Y.P., Wong, A.S.W., Wong, T., Chung, J. and Newton, E. (2005) Effects of Wearing N95 and Surgical Facemasks on Heart Rate, Thermal Stress and Subjective Sensations. International Archives of Occupational and Environmental Health, 78, 501-509. http://dx.doi.org/10.1007/s00420-004-0584-4

[4] Farquharson, C. and Baguley, K. (2003) Responding to the Severe Acute Respiratory Syndrome (SARS) Outbreak: Lessons Learned in a Toronto Emergency Department. Journal of Emergency Nursing: JEN: Official Publication of the Emergency Department Nurses Association, 29, 222-228.

[5] Lim, E.C.H., Seet, R.C.S., Lee, K.H., Wilder-Smith, E.P.V., Chuah, B.Y.S. and Ong, B.K.C. (2006) Headaches and the N95 Face-Mask amongst Healthcare Providers. Acta Neurologica Scandinavica, 113, 199-202. http://dx.doi.org/10.1111/j.1600-0404.2005.00560.x

[6] Hayashi, C. and Tokura, H. (2004) The Effects of Two Kinds of Mask (with or without Exhaust Valve) on Clothing Microclimates inside the Mask in Participants Wearing Protective Clothing for Spraying Pesticides. International Archives of Occupational and Environmental Health, 77, 73-78. http://dx.doi.org/10.1007/s00420-003-0472-3

[7] Roberge, R.J., Bayer, E., Powell, J.B., Coca, A., Roberge, M.R. and Benson, S.M. (2010) Effect of Exhaled Moisture on Breathing Resistance of N95 Filtering Facepiece Respirators. Annals of Occupational Hygiene, 54, 671-677. http://dx.doi.org/10.1093/annhyg/meq042

[8] Grützenmacher, S., Lang, C., Mlynski, R., Mlynski, B. and Mlynski, G. (2005) Long-Term Rhinoflowmetry: A New Method for Functional Rhinologic Diagnostics. American Journal of Rhinology, 19, 53-57.

[9] Bałazy, A., Toivola, M., Adhikari, A., Sivasubramani, S.K., Reponen, T. and Grinshpun, S.A. (2006) Do N95 Respirators Provide 95\% Protection Level against Airborne Viruses, and How Adequate Are Surgical Masks? American Journal of Infection Control, 34, 51-57. http://dx.doi.org/10.1016/j.ajic.2005.08.018 